\title{
Comparison of optical reflectance spectrum at blade and vein parts of cabbage and kale leaves
}

\author{
Viet-Duc Ngo ${ }^{1}$, Dong-Ki Ryu ${ }^{1}$, Sun-Ok Chung ${ }^{1 *}$, Sang-Un Park ${ }^{2}$, Sun-Ju Kim ${ }^{3}$, Jong-Tae Park ${ }^{4}$ \\ ${ }^{1}$ Department of Biosysytem Machine Engineering, Chungnam National University, Dajeon 305-764, Korea, ${ }^{2}$ Department of crop scienc, Chungnam \\ National University, Dajeon 305-764, Korea, ${ }^{3}$ Department of Bio Environmental Chemistry, Chungnam National University, Dajeon 305-764, \\ Korea, ${ }^{4}$ Department of Food Science and Technology Chungnam National University, Dajeon 305-764, Korea
}

Received on 30 December 2012, revised on 10 June 2013, accepted on 10 June 2013

\begin{abstract}
Objective of the study was to compare reflectance spectrum in the blade and the vein parts of cabbage and kale leaves. A total 6 cabbage and kale leaves were taken from a plant factory in Chungnam National University, Korea. Spectra data were collected with a UV/VIS/NIR spectrometer (model: USB2000, Ocean Optics, FL, USA) in the wavelength region of $190-1130 \mathrm{~nm}$. Median filter smoothing method was selected to preprocess the obtained spectra data. We computed reflectance difference by subtraction of averaged spectrum from individual spectrum. To estimate correlation at different parts of cabbage and kale leaves, cross - correlation method was used. Differences between cabbage and kale leaves are clearly manifested in the green, red and near - infrared ranges. The percent reflectance of cabbage leaves in the NIR wavelength band was higher than that of kale leaves. Reflectance in the blade part was higher than in the vein part by $18 \%$. Reflectance difference in the different parts of cabbage and kale leaves were clear in all of the wavelength bands. Standard deviation of reflectance difference in the vein part was greater for kale, while the value in the blade part was greater for cabbage leaves. Standard deviation of cross - correlation increased from 0.092 in the first sensor (UV/VIS) and 0.007 in the second sensor (NIR) to 0.099 and 0.015 , respectively.
\end{abstract}

Key words : Cabbage, Kale, Reflectance, Spectrometer, Reflectance Difference

\section{Introduction}

Remote sensing techniques are well-established non-instructive tools in assessing changes in the structure and function of ecosystems. Remote sensing of the optical properties of different cultural plant species subjected to stress factors gives the possibility to distinguish symptoms below the subjective level of detection and timely to mitigate the risk of their action. The spectral characteristics of vegetation are determined mainly by scattering and reflectance characteristics of the internal leaf structure and biochemical constituents (Krezhova et al., 2005).

A green leaf contributes photosynthesizing and yields a typical healthy green reflectance spectra with

\footnotetext{
*Comesponding author: Tel: +82-42-821-6712

E-mail address: sochung@cnu.ac.kr
}

strong leaf pigments in the blue and red regions, and a peak in reflectance in the green region of the visible spectrum. The high diffuse reflectance of the near infrared (700 - $1200 \mathrm{~nm}$ ) energy from plant leaves is due to the internal scattering at the cell wall - air interfaces within the leaf. A water vapor absorption band exists at $920-980 \mathrm{~nm}$. Therefore, the optimum spectral region for sensing in the near - infrared region is believed to be $740-900 \mathrm{~nm}$ (Jensen, 2000). Several studies have related spectral reflectance with different crops (Gates et al., 1995; Liu et al., 2011) evaluated the effect of difference in crop species on singularity parameters. They selected rice, maize, soybean and cabbage at mature growth stage. They found that the spectral reflectance of different species at a same growth stage showed different percentages of reflectance. The difference between cabbage and 
kale leaves can be used to distinguish if the difference between the spectral reflection in certain wavelength regions from different plants can be recognised (Zwiggelaar, 1998).

Objective of the paper was to compare reflectance spectra in different parts between Chinese cabbage and kale leaves in visible and near - infrared wavelength bands.

\section{Materials and Method}

\section{Cabbage and kale samples}

Chinese cabbage and kale samples were taken from a plant factory in Chungnam National University, Korea. The number of samples used in spectra measurements were 6 including 3 cabbage leaves and 3 kale leaves. The samples were planted and cultivated under a 16/8-h day/night cycle and the same conditions of light source (Red : Blue : White = $11: 4: 3), \mathrm{CO}_{2}(1000$ $\pm 100 \mathrm{ppm})$, temperature $\left(20 \pm 1^{\circ} \mathrm{C}\right)$, humidity $(65 \pm$ $5 \%), \mathrm{pH}(6.0 \pm 5)$ and (EC) electrical conductivity $(1,200 \pm 90 \mu \mathrm{S} / \mathrm{cm})$. The samples selected for spectra measurements were healthy.

\section{Spectra measurements}

Reflectance spectra were measured at evenly distributed 18 points ( 9 points in each blade and vein parts)

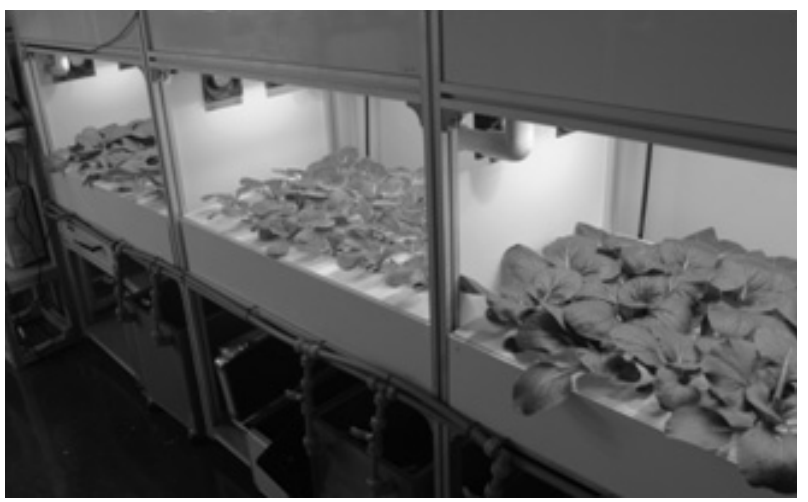

Fig. 1. Chinese cabbage and kale planted in a plant factory. using a Jaz spectrometer (model: USB2000, Ocean Optics, FL, USA) in a dark external condition. Fig. 2 shows photo of leaf reflectance measurement using the spectrometer. The measured leaves did not remove from the plants. A light source constructed with a Deuterium - Tungsten Halogen (200 - 1000 nm) lamp was used for the spectra measurement. The spectrometer was consisted of two CCD detectors with the measurement ranges of $190-890 \mathrm{~nm}$ (UV/VIS) and $470-$ $1130 \mathrm{~nm}$ (NIR), respectively. The resolution of reflectance spectra was about $0.38 \mathrm{~nm}$. The spectra were collected using the software provided by the manufacturer. The spectra in the range of $400-1050 \mathrm{~nm}$ were used to build reflectance difference and correlation models due to excessive noise in other wavelength ranges. The spectra measured at the 9 points on each part of each sample were averaged and then the mean spectrum was used as a representative spectrum of each sample leaf.

\section{Data analysis}

To reduce the noise, the original spectral data were preprocessed by median filter method with a 7 segment size using the Unscrambler (ver. 9.7, CAMO Process AS, Norway) software package based on preliminary data analysis and results suggested by Suh et al. (2012). Reflectance difference was computed by subtraction

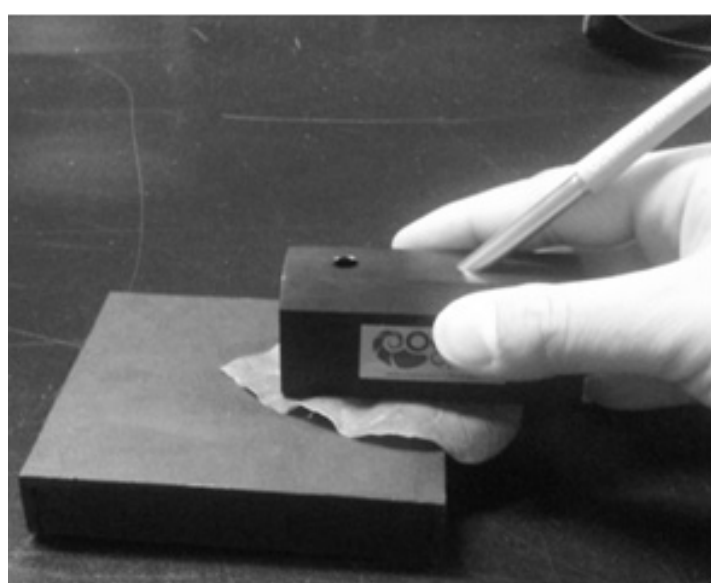

Fig. 2. Photo of leaf measurement using the spectrometer. 
of averaged spectrum from each spectrum. Cross correlation is a standard method of estimating the degree to which two series are correlated. A correlation analysis between the two parts of cabbage and kale leaves was computed by cross - correlation method using Matlab 7.10 (Mathworks, California, USA) to determine if the reflectance at each part of cabbage leaf was positively or negatively correlated with each part of kale leaf.

\section{Results and Discussion}

\section{Leaf reflectance}

Reflectance spectra of leaves between 400 - 1050 $\mathrm{nm}$ measured for cabbage and kale leaves are shown in Fig. 3. Percent reflectance of kale and cabbage leaves in visible wavelength (VIS) were quite different. As it could be seen, the differences between cabbage and kale leaves were clearly manifested within 520 $580 \mathrm{~nm}$, and $690-800 \mathrm{~nm}$ ranges. The highest reflectance of kale leaves in VIS wavelength were about $10 \%$ and $12 \%$ at the blade and vein parts, respectively, while the highest of cabbage leaves were about $5 \%$ at both of the blade and vein parts. However, the percent reflectance of cabbage leaves in NIR wavelength band was higher than that of kale leaves. Reflectance in the blade part was also higher than in the vein part by $18 \%$.

\section{Reflectance difference}

The reflectance difference for each wave-band was computed between averaged reflectance and each reflectance as in the following equation (Hu et al., 2010).
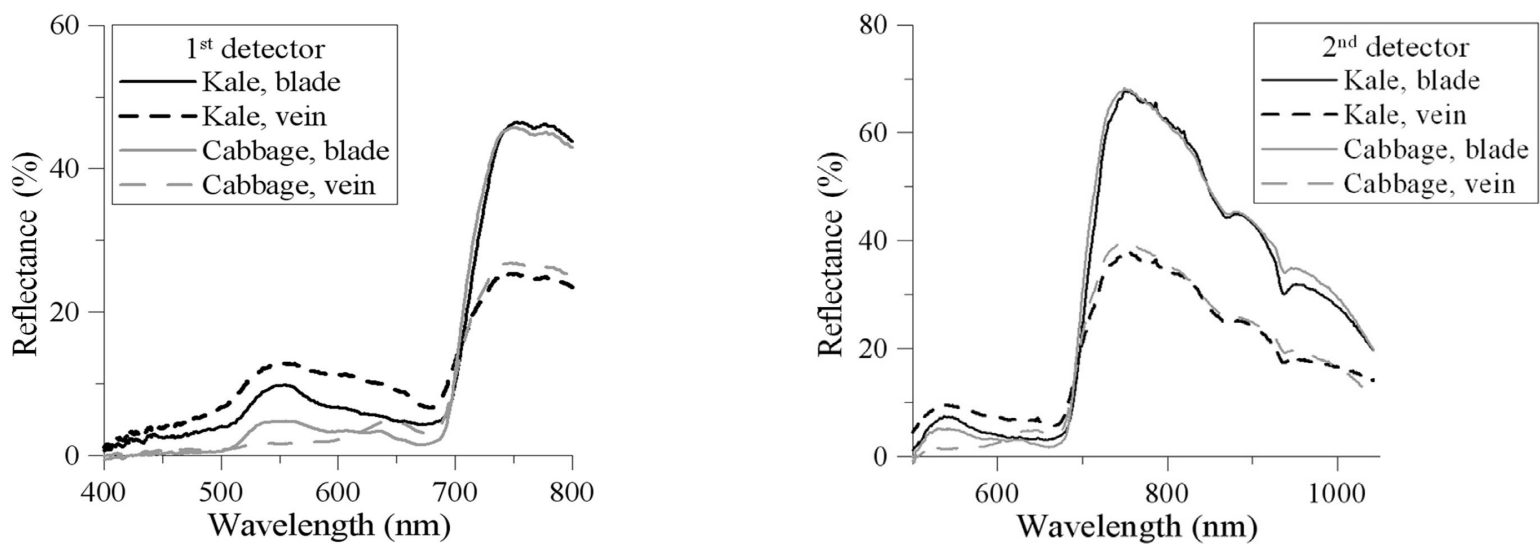

Fig. 3. Percentage reflectance of leaves measured by the spectrometer.
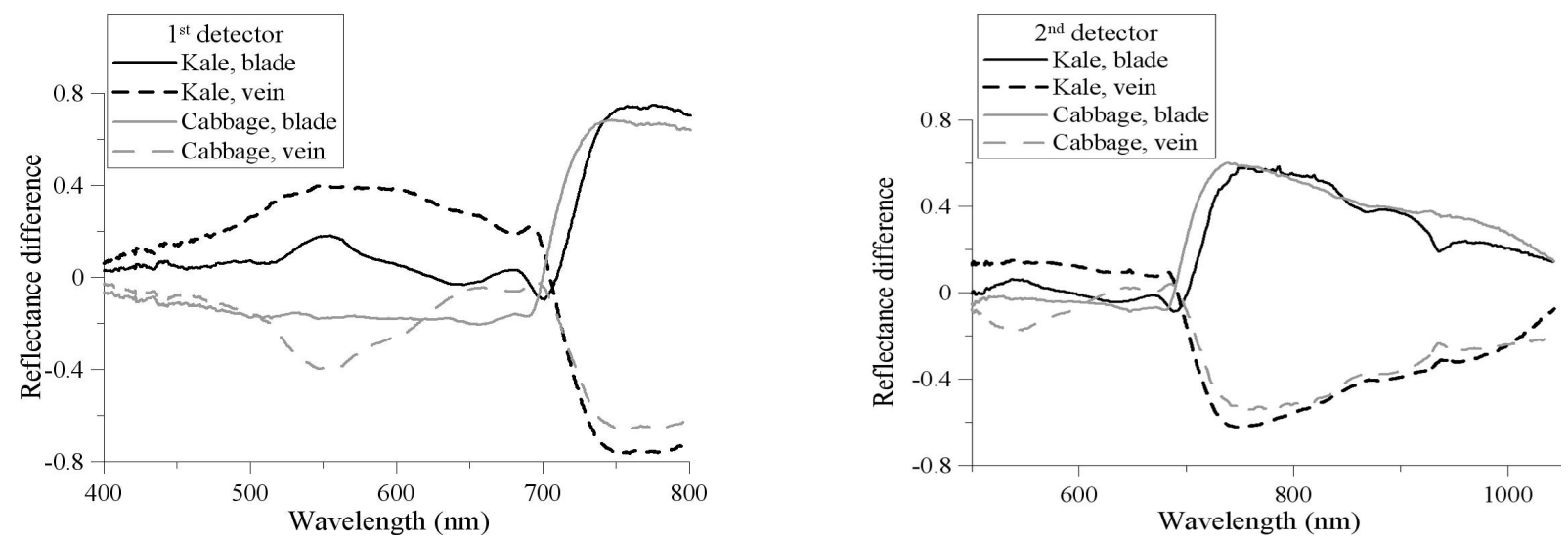

Fig. 4. Reflectance difference in the VIS and NIR wavelength bands. 

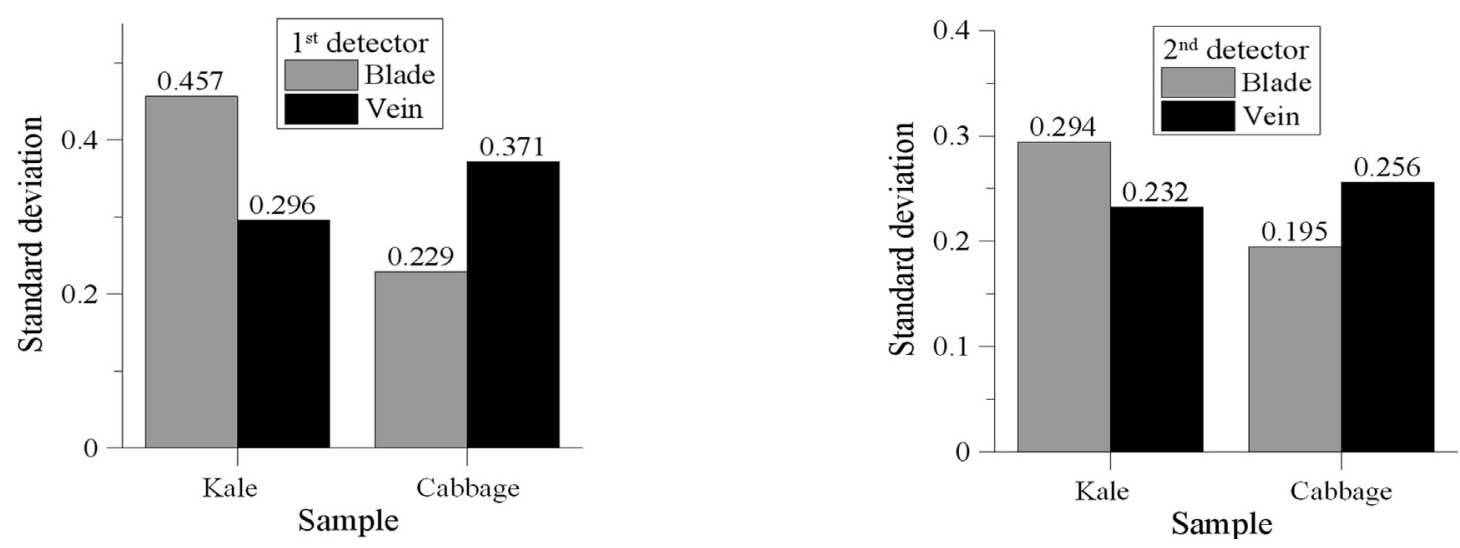

Fig. 5. Standard deviation of reflectance difference in the VIS and NIR bands.

Reflectance Difference $=\frac{\text { Reflectance }- \text { Averaged Reflectance }}{\text { Meanof Averaged Reflectance }}$

Fig. 4 shows the difference reflectance in the different parts of cabbage and kale leaves for the two detectors. In general, reflectance difference in different parts of cabbage and kale leaves was clear in both of the wavelength bands with the highest different values of 0.4 and 0.75 in the VIS and NIR wavelength bands, respectively. Especially in the NIR wavelength band, it was clear that reflectance in the blade part and in the vein part was similar for cabbage and kale, while reflectance in the VIS wavelength band was quite different. Great difference could be identified visually with VIS wavelength band, especially at $550 \mathrm{~nm}$ wavelength.

Reflectance difference could be shown clearer when we computed standard deviation of reflectance difference as shown in Fig. 5. In both of two wavelength bands, standard deviation of reflectance difference in the vein part was greater for kale leaves, while standard deviation in the blade part was greater for cabbage leaves.

\section{Cross - correlation}

Cross - correlation in a range of $700-850 \mathrm{~nm}$ was obtained with the data from the first detector, and

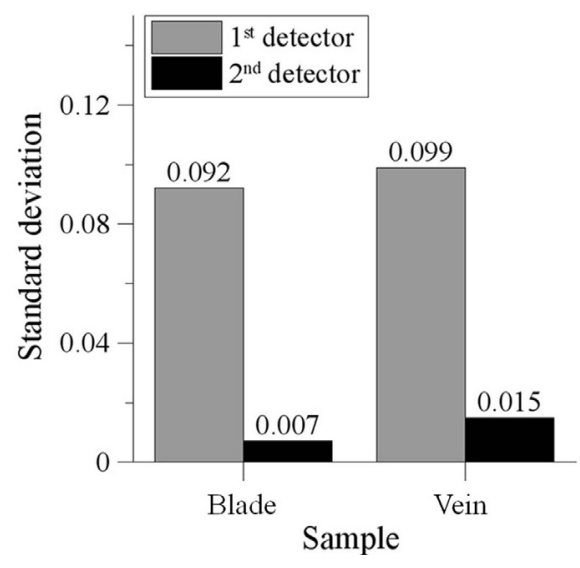

Fig. 6. Standard deviation of cross - correlation of reflectance difference for the blade and vein parts.

700 - $1050 \mathrm{~nm}$ with second sensor. After cross correlation was computed, standard deviation of cross correlation also computed and displayed in Fig. 6. Overall, standard deviation of cross - correlation increased from 0.092 and 0.007 of blade part in the first and second sensors to 0.099 and 0.015 of vein part, respectively.

\section{Conclusion}

Overall, the results of the reflectance measurement indicate that there is difference in VIS wavelength band between Chinese cabbage and kale leaves. In the NIR wavelength band, however, the reflectance difference was lower in the same parts of both species. Standard deviation decreased from kale leaves to cabbage leaves 
in the blade part while it increased in the vein part. Comparisons of reflectance difference and cross correlation showed low value of standard deviation. From the results of this study, we suggested that reflectance could be similarly obtained in the blade and vein parts while standard deviation of blade and vein was different by not more than $20 \%$. Using cross correlation method, the reflectance correlation could be obtained for comparison between Chinese cabbage leaves and kale leaves at some of certain wavelength bands. Moreover, this method offered possibilities to compare leaf growth of different species, different cultivating conditions using optical reflectance of leaf. This study could be further used for estimation of crop condition and estimation of leaf pigment content, however, further research would be needed to investigate effects of leaf surface, light source, $\mathrm{pH}, \mathrm{EC}$, temperature, humidity, and $\mathrm{CO}_{2}$.

\section{Acknowledgment}

This research was supported by Korea Institute of Planning and Evaluation for Technology in Food, Agriculture, Forestry and Fisheries (Project No. 311022-
05-2-SB030), Ministry for Food, Agriculture, Forestry and Fisheries, Republic of Korea.

\section{References}

Gates DM, Keegan HJ, Schleter JC, Weidner VR. 1965. Spectral properties of plants. Applied Optics 4:11-20.

Hu CG, Sun LD, Flores-Camacho JM, Hohage M, Liu CY, Hu XT. 2010. A rotating-compensator based reflectance difference spectrometer for fast spectroscopic measurements. Review of Scientific Instruments 81 (043108):1-11.

Jensen JR. 2000. Remote Sensing of the Environment An Earth Resource Perspective. Prentice-Hall, Upper Saddle River, New Jersey.

Krezhova DD, Yanev TK, Alexieva VS, Ivanov SV. 2005. Early detection of changes in leaf reflectance of pea plants (Pisum sativum L.) under herbicide action. Recent Advances in Space Technologies: Proceedings of 2nd International Conference on Recent Advances in Space Technologies. pp. 636-641. Military Museum Istanbul, Turkey.

Liu M, Liu X, Wu L, Duan L, Zhong B. 2011. Wavelet-based detection of crop zinc stress assessment using hyperspectral reflectance. Computer and Geosciences 37:1254-1263.

Suh SR, Lee KH, Yu SH, Shin HS, Choi YS, Yoo SN. 2012. A melon fruit grading machine using a miniature VIS/NIR spectrometer: 1. Calibration models for the prediction of soluble solids content and firmness. Journal of Biosystems Engineering 37:166-176.

Zwiggelaar R. 1998. A review of spectral properties of plants and their potential use for crop/weed discrimination in row-crops. Crop Protection 1:189-206. 